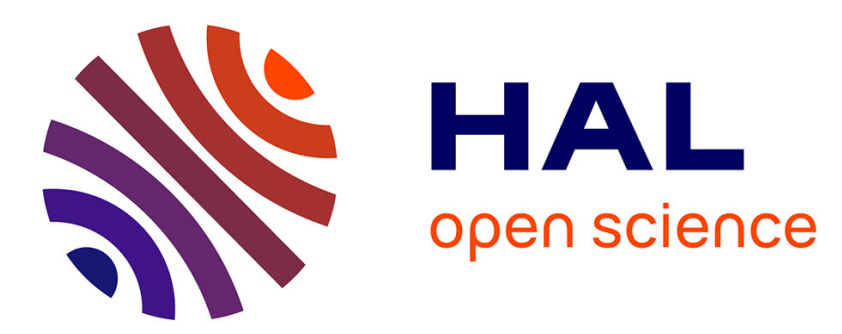

\title{
6. Effet de plusieurs bandes d'énergie interdite sur les structures de phonons dans des expériences tunnel
}

\author{
J. Klein, A. Léger
}

\section{To cite this version:}

J. Klein, A. Léger. 6. Effet de plusieurs bandes d'énergie interdite sur les structures de phonons dans des expériences tunnel. Revue de Physique Appliquée, 1970, 5 (6), pp.901-902. 10.1051/rphysap:0197000506090103 . jpa-00243476

\section{HAL Id: jpa-00243476 https://hal.science/jpa-00243476}

Submitted on 1 Jan 1970

HAL is a multi-disciplinary open access archive for the deposit and dissemination of scientific research documents, whether they are published or not. The documents may come from teaching and research institutions in France or abroad, or from public or private research centers.
L'archive ouverte pluridisciplinaire HAL, est destinée au dépôt et à la diffusion de documents scientifiques de niveau recherche, publiés ou non, émanant des établissements d'enseignement et de recherche français ou étrangers, des laboratoires publics ou privés. 


\section{EFFET DE PLUSIEURS BANDES D'ÉNERGIE INTERDITE SUR LES STRUCTURES DE PHONONS DANS DES EXPÉRIENCES TUNNEL}

\section{J. KLEIN et A. LÉGER}

Groupe de Physique des Solides de l'E. N. S., Faculté des Sciences,

Tour 23, Paris 5e, France

Il est bien connu que les caractéristiques tunnel de jonctions faites avec des films épais de $\mathrm{Pb}$ montrent deux gaps bien définis [1]. Néanmoins nous avons montré expérimentalement que sur des jonctions $\mathrm{Al}-\mathrm{I}-\mathrm{Pb}$ il n'existe pas un décalage correspondant des pics de phonons. A l'aide des équations d'Eliashberg nous avons essayé d'expliquer ce problème. C'est la partie imaginaire de la fonction complexe du gap qui donne les structures de phonons dans les courbes d'effet tunnel. Cette partie imaginaire peut 
être calculée au voisinage d'un point critique de la densité de phonons en supposant un double gap. Nous obtenons ainsi une expression très voisine de celle obtenue par Schrieffer et al. [2] mais avec une densité effective de phonons qui est la somme de deux fonctions : la première est centrée à $\hbar \omega_{\mathrm{Ph}}+\Delta_{1}$ et la seconde à $\hbar \omega_{\mathrm{Ph}}+\Delta_{2}$. Nous avons calculé cette densité effective de phonons à partir des résultats de Bennett [3] et nous obtenons un seul extremum. Ceci peut expliquer le fait que dans une jonction tunnel exhibant un double gap, il n'y a pas de décalage de la structure des phonons.

\section{Bibliographie}

[1] Voir par exemple : ToMASch (W. J.), Phys. Rev. Letters, 1965, 15, 672.

BlaCkFord (B. L.), MARCH (R. H.), Phys. Rev., 1969, 18b, 397.

[2] Scalapino (D. J.), Schrieffer (J. R.), Wilkins (J. W.), Phys. Rev., 1966, 148, 263.

[3] Bennett (A. J.), Phys. Rev., 1965, 140A, 1902. 